\title{
New Method for Calculating Bottom Hole Pressure of Horizontal Well in Fracture-cavity Reservoir Based on Point Source Function
}

Hailong Liu ( $\sim$ 478277608@qq.com )

sinopec

\section{Research Article}

Keywords: point source function, horizontal well, fracture-vuggy reservoir, bottom hole pressure, fracture

Posted Date: July 29th, 2021

DOl: https://doi.org/10.21203/rs.3.rs-658336/v1

License: (c) (1) This work is licensed under a Creative Commons Attribution 4.0 International License.

Read Full License 
A new method for calculating bottom hole pressure of horizontal well in fracture-cavity reservoir based on point source function

$$
\text { Liu Hailong } 1,2
$$

1. China University of Geosciences (Beijing), Haidian District, Beijing 100083, China;

2. Northwest Center, Sinopec Petroleum Exploration and Development Research

$$
\text { Institute, Haidian District, Beijing 100083, China }
$$

Abstract: Accurate determination of unsteady bottom hole pressure helps to monitor and predict well production in real-time. On the premise of fully considering the seepage characteristics of carbonate rock, a new source function suitable for the seepage of carbonate rock is established. It enlarges the application scope of source function theory and lays a theoretical foundation for solving the seepage problem of carbonate rock. This paper presents the process of solving bottom hole pressure step by step.

Step 1: Based on the triple media model, the Pedrosa permeability calculation formula is applied to establish the seepage model of the triple media reservoir considering the formation stress sensitivity.

Step 2: By perturbation transform and Laplace transform, the point source function considering stress sensitivity in carbonate reservoir is obtained in Laplace space. The point source function in the infinite plate reservoir is obtained by the principle of mirror image and superposition.

Step 3: The method of solving the horizontal well pressure under the constant pressure boundary is established.

Through literature comparison and numerical simulation, the rationality of the proposed method is verified. Simultaneously, the sensitivity analysis of pressure and pressure derivative is carried out, and the influences of fracture number, fracture angle, fracture half-length, skin factor, horizontal well segment length, and horizontal well segment spacing on pressure and pressure derivative are analyzed in detail. Considering fracture orientation and stress sensitivity, we divide the triple media fracture-vuggy reservoir fluid flow into five stages. The number of fractures and fracture direction mainly affect stage C. In contrast, the length of horizontal subsection and skin factor mainly affect stage B. Stage D is more obvious when the fracture half-length and the horizontal 
sublevel interval of the horizontal well are small.

Key words: point source function, horizontal well, fracture-vuggy reservoir, bottom hole pressure, fracture

\section{Introduction}

Source function is one of the most important methods to solve the problem of reservoir seepage. In 1963, Warren first introduced source function into the field of reservoir seepage (Warren, 1963). In 1973, Ramey further extended the application range of source function and established point source function, line source function, and surface source function under different reservoir boundary conditions (Ramey, 1973, 1974). In 1987, Kuchuk and Ozkan applied Laplace transform to establish point source function, line source function, and surface source function to solve the seepage problem of the dual-medium reservoir under different reservoir boundaries (Kuchuk, 1987; Ozkan, 1987). These above source functions are suitable to solve the reservoir seepage problems in vertical wells, horizontal wells, multi-branch wells, and fractured horizontal wells. What's more, they can provide an important theoretical basis for oil well productivity prediction, well test interpretation, and understanding the development laws of different well types. However, with the development of carbonate rocks, the traditional source function's application is greatly limited. Carbonate rock is a triple media reservoir with a certain stress-sensitive effect. Still, the existing source function can not consider the influence of stress sensitivity on the seepage of the oil reservoir in triple media.

As for bottom hole pressure solution, domestic and foreign scholars have done a lot of research and made great progress. In 1993, Onur used the typical curve method to solve the analytical solution of bottom hole pressure for a naturally fractured reservoir (Onur, 1993). In 1999, Zhang approximately treated the fractured vertical well as a single fracture and derived the analytical solution of the bottom hole pressure of the fractured vertical well (Zhang, 1999). In 2000, Bui used the Laplace transform to calculate the bottom hole pressure of a fractured vertical well for a partially perforated reservoir (Bui, 2000). In 2001, Song et al. solved the bottom hole pressure of steady-state fractured vertical well by using the method of separation of variables 
according to the two-parameter continuous model and carried out sensitivity analysis (Song, 2001). In 2003, using the three-parameter continuous model and the mean mass conservation method, Deng et al. gave the steady-state and unsteady-state pressure solution for vertically fractured wells (Deng et al. 2003). In 2005, based on previous studies, Li et al. provided the analytical solution of bottom hole pressure of vertically fractured wells in tight sandstone reservoirs through approximate and modified methods (Li et al. 2005). In 2008, Zhang et al. provided a revised numerical solution of bottom hole pressure based on the rock body stress equation (Zhang et al. 2008). In 2010, Zhang et al. deduced bottom hole pressure of vertical well by considering the pressure-sensitive effect and start-up pressure (Zhang et al. 2010). In 2011, Zhang et al. carried out productivity analysis of fractured vertical wells based on pressure distribution equation and elastic productivity variation law (Zhang et al. 2011). In 2012, Stalgorova and Matter used finite element and discrete numerical methods to solve bottom hole pressure numerical solutions for horizontal wells under different reservoir conditions (Stalgorova and Matter, 2012, 2013). In 2013, by taking non-Darcy seepage in tight sandstone reservoirs into consideration, Jiang et al. treated hydraulic fracturing fractures as finite conductivity fractures. They derived the numerical solution of bottom hole pressure in unsteady fractured vertical wells (Jiang et al., 2013). In 2014, Wang et al. established a steady-state pressure model of a long-fracture fractured vertical well using bilinear seepage and conformal transformation method (Wang et al. 2014). In 2015, Zhang et al. studied the fluid flow rule in a tight sandstone reservoir and considered the deformation coefficient and start-up pressure, and they obtained the pressure analytical solution of the fractured vertical well (Zhang et al. 2015).

Literature survey found that although there are many ways to solve bottom hole pressure, we can summarize them in three aspects:

Single media pressure equation: Based on Darcy's law, the relationship between productivity and pressure difference is established by considering linear or radial fluid flow. Generally, the relationship between the two is linear.

Dual medium pressure equation: Consider the fracture and matrix and the channeling flow between the fracture and matrix. The pressure equation is obtained 
according to the equal pressure and flow at the intersection of the fracture and the matrix interface.

Triple medium pressure equation: Considering the fluid flow in the fracture, matrix, and karst cave, based on the positional relationship of the well, karst cave, and fracture, the pressure and flow rate at the intersection of the fracture, matrix, and karst cave is equal to obtain the pressure distribution.

Although the influence of fractures on bottom hole pressure is considered in the literature, the orientation of fractures is generally treated as simple, such as fractures parallel to each other or perpendicular to the horizontal section of the horizontal well. Fractures are of various orientations and sizes, and skin factor varies from fracture to fracture. Therefore, it is necessary to consider the actual situation of fracture and establish a pressure-solving model. This paper presents a new way to obtain the point source function. Firstly based on the triple media model, the Pedrosa permeability calculation formula is applied to establish the triple media reservoir seepage model considering the formation stress sensitivity. Secondly, by perturbation transform and Laplace transform, the point source function considering stress sensitivity in carbonate reservoir is obtained in Laplace space. Then, the point source function of the infinite plate fracture-cavity reservoir is obtained by the mirror image and superposition principle. Based on the new point source function, this paper presents "three steps" to solve the pressure of a horizontal well under the constant pressure boundary.

\section{Model establishing}

\subsection{Physical model}

The carbonate reservoirs develop fractures, and the distribution of fractures is complex, and many karst caves are forming along with the fractures. The carbonate reservoir has a triple medium property consisting of natural fractures, karst caves, and matrix. In the development of carbonate reservoirs, if the production well does not drill the cave, hydraulic fracturing must build the artificial fractures and communicate with the caves and the natural fractures in the matrix.

Fractured-vuggy reservoirs have diverse reservoir spaces and complex fluid flow. The actual fluid flow diagram in the reservoir is shown in figure 1 (a). To simplify 
calculations and facilitate mathematical modeling, the basic flow of the reservoir is simplified as the flow shown in figure 1 (b). Meanwhile, the following assumptions are made:

(1) The oil reservoir is composed of the cave system, matrix system, and fracture system. The cave and matrix system are the main storage space, and the fracture system is the main flow channel. All the well production comes from the influx of the fracture system;

(2) Considering the permeability sensitivity of natural fracture system, it is assumed that the permeability of matrix system is constant;

(3) There is a point source in the oil reservoir. The initial pressure is equal everywhere in the oil reservoir, and the reservoir temperature is constant during the production process of the oil well.

(4) The reservoir fluid is slightly compressible, and the influence of gravity and capillary force is not considered. The porosity of the reservoir and fluid viscosity is constant.

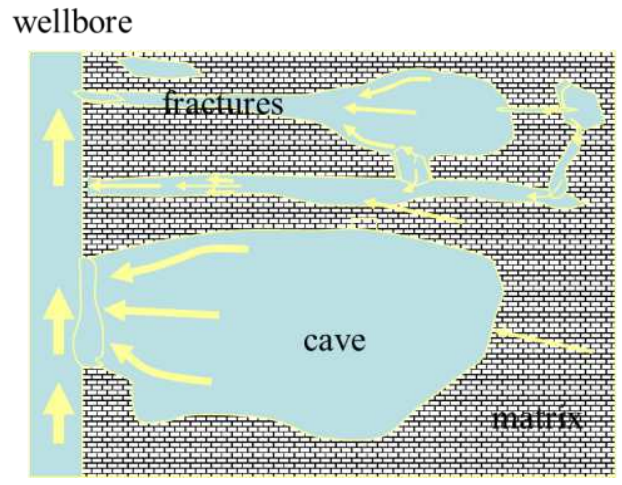

(a) Actual fluid flow

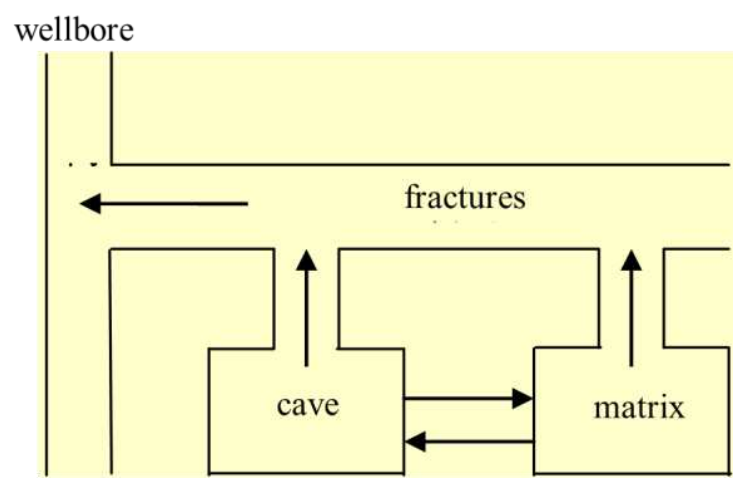

(b) Simplified fluid flow

Figure 1 Simplified diagram of fluid flow

Since the orientation of fractures is not uniform, the number of fractures in the reservoir is assumed to be $\mathrm{m}$, and the horizontal section of the horizontal well is divided into $\mathrm{m}$ sections accordingly. The center of each horizontal well segment is parallel to the fracture center along the z-axis. After division, the schematic diagram is shown in figure 2. Without considering the channeling between fractures, each fracture can be regarded as the source phase of the corresponding horizontal well segment. Then, considering the fluid flow of a fracture and the related horizontal section, the pressure model under a 
single fracture can be established. Finally, the pressure distribution of the entire horizontal well can be obtained according to the pressure superposition.

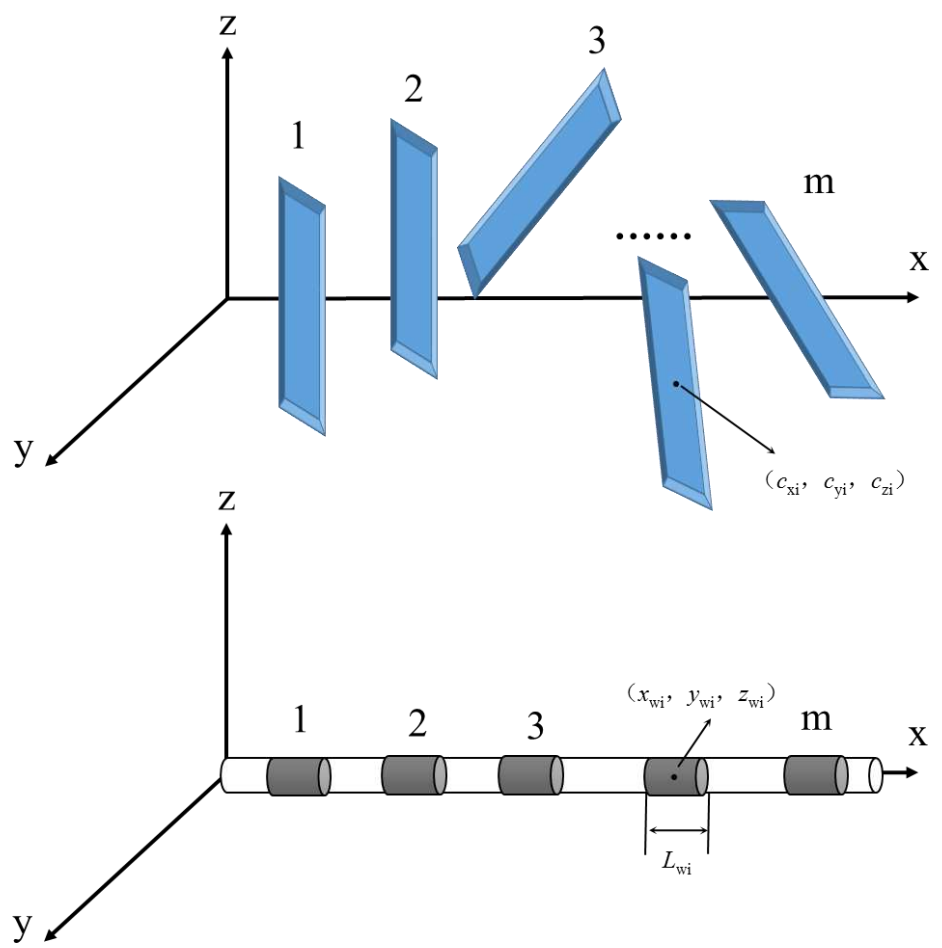

Figure 2 Schematic diagram of fracture distribution and horizontal section division of horizontal wells

\subsection{Mathematical model}

Based on the above assumptions, the center of a single fracture is located at $\left(\mathrm{x}_{0}, \mathrm{y}_{0}\right.$, $\mathrm{z}_{0}$ ), and the governing equation of the fracture is shown as follows:

$$
\begin{aligned}
& \frac{k_{x}}{\mu} \frac{\partial^{2} p_{f}}{\partial x^{2}}+\frac{k_{y}}{\mu} \frac{\partial^{2} p_{f}}{\partial y^{2}}+\frac{k_{z}}{\mu} \frac{\partial^{2} p_{f}}{\partial z^{2}}+\frac{8 q w_{x} w_{y} w_{z}}{\mu} f(x, y, z) \\
& =(\phi c)_{f} \frac{\partial p_{f}}{\partial t}+(\phi c)_{m} \frac{\partial p_{m}}{\partial t}+(\phi c)_{v} \frac{\partial p_{v}}{\partial t}
\end{aligned}
$$

$f(x, y, z)$ is the source (sink) phase, and its expression is shown as below.

$$
\begin{gathered}
f(x, y, z)=\frac{1}{8 w_{x} w_{y} w_{z}} \iiint_{v} \delta\left[\left(x-x_{0}\right)\left(y-y_{0}\right)\left(z-z_{0}\right)\right] d v \\
\left(x_{0}-w_{x}\right)\left(y_{0}-w_{y}\right)\left(z_{0}-w_{z}\right) \leq v \leq\left(x_{0}+w_{x}\right)\left(y_{0}+w_{y}\right)\left(z_{0}+w_{z}\right)
\end{gathered}
$$

Where $\delta$ is the Dirac Delta function.

If $\Delta p=p_{i}-p$, then the change trend of $\Delta p$ and $p$ is consistent. The pressure term $p$ in the governing equation (1) can be directly replaced with pressure difference term $\Delta p$. 


$$
\begin{aligned}
& \frac{k_{x}}{\mu} \frac{\partial^{2} \Delta p_{f}}{\partial x^{2}}+\frac{k_{y}}{\mu} \frac{\partial^{2} \Delta p_{f}}{\partial y^{2}}+\frac{k_{z}}{\mu} \frac{\partial^{2} \Delta p_{f}}{\partial z^{2}}+\frac{8 q w_{x} w_{y} w_{z}}{\mu} f(x, y, z) \\
& =(\phi c)_{f} \frac{\partial \Delta p_{f}}{\partial t}+(\phi c)_{m} \frac{\partial \Delta p_{m}}{\partial t}+(\phi c)_{v} \frac{\partial \Delta p_{v}}{\partial t}
\end{aligned}
$$

Similarly, the governing equation of the matrix system can be obtained as the equation (5)

$$
(\phi c)_{m} \frac{\partial \Delta p_{m}}{\partial t}=\sigma_{m} \frac{k_{m}}{\mu}\left(\Delta p_{f}-\Delta p_{m}\right)
$$

The governing equation of the karst cave system is obtained as the equation (6)

$$
(\phi c)_{v} \frac{\partial \Delta p_{v}}{\partial t}=\sigma_{v} \frac{k_{v}}{\mu}\left(\Delta p_{f}-\Delta p_{v}\right)
$$

Where, $k_{x}, k_{y}$ and $k_{z}$ are the permeability in $\mathrm{x}, \mathrm{y}$ and $\mathrm{z}$ directions, $\mathrm{mD} ; \mu$ is the viscosity of crude oil, mPa.s; $\Delta \mathrm{p}$ is pressure difference, $\mathrm{MPa} ; \phi$ is the porosity, decimal; $\mathrm{c}$ is the compressibility coefficient, $\mathrm{Pa}^{-1}$; Subscript "m" is the matrix system; Subscript "f" is natural fracture system; Subscript "v" refers to the karst cave system.

When considering the stress sensitivity of the natural fracture system, the natural fracture permeability decreases with the decrease of formation pressure. The natural fracture permeability can be expressed as:

$$
k_{x}=k_{x i} e^{-\alpha\left(p_{i}-p\right)}=k_{x i} e^{-\alpha \Delta p}
$$

Where $\mathrm{k}_{\mathrm{xi}}$ is the initial permeability of the natural fracture system in the $\mathrm{x}$ direction, $10^{-3} \mu^{2}$; $\mathrm{p}_{\mathrm{i}}$ is the initial formation pressure, $\mathrm{MPa} ; \alpha$ is the permeability modulus, $\mathrm{MPa}^{-1}$ 。

\section{Model solving}

\subsection{Solution research}

Literature survey found that there are four main methods to solve the equation (4). First: assuming that the reservoir pressure reaches the bottom of the well instantaneously, based on the Green's function, the analytical solution of the pressure in the Laplace space domain is obtained (Bui, 2000; Li et al. 2005; Zhang et al.2011); Second: a numerical solution to the pressure in the real domain is obtained based on the separation of variables and the numerical difference discrete method. (Zhang et al. 1999; Stalgorova and matter, 2012, 2013); Third: Assume that the bottom hole flow reaches a pseudo-steady state, ignore the pressure and time changes, only consider the 
relationship between pressure and space, and use the successive steady-state method to obtain the pressure distribution (Brown et al.,1987; Onur and Satman, 1993; Zhang et al. 2008); Fourth: get the pressure in the Laplace space through dimensionless change, Laplace transform, and Fourier transform and then obtain the real domain pressure numerical solution through numerical inversion (Jiang et al. 2103; Sureshjani and Clarkson, 2015; Liu, 2016). This paper considers the triple-media and stress-sensitive characteristics of fracture-cavity reservoirs, applying perturbation transformation, Laplace transforms, mirror image principle and Poisson summation formula, a new point source function for fracture-cavity reservoirs is established. Simultaneously, considering the problem that the conventional source function cannot calculate the formation stress sensitivity, the numerical solution of the bottom hole pressure of the horizontal well is solved.

\subsection{Dimensionless transformation}

The dimensionless transformation is defined as follows:

$$
\begin{gathered}
t_{D}=\frac{N=\sqrt{\frac{a b h}{L}}}{(\phi \mu c)_{f+m+v} N^{2}} t \quad P_{D j}=\frac{2 \pi k_{i f} N\left(p_{i}-p_{j}\right)}{Q B \mu}, j=f / m \quad k_{i f}=\sqrt[3]{k_{f r}^{2} k_{f z}} \\
r_{D}=\sqrt{\left[\left(\sqrt{\frac{k}{k_{x}}} \frac{x}{N}\right)^{2}+\left(\sqrt{\frac{k}{k_{y}}} \frac{y}{N}\right)^{2}+\left(\sqrt{\frac{k}{k_{z}}} \frac{z}{N}\right)^{2}\right]} \\
a_{D}=\frac{a}{N} \quad b_{D}=\frac{b}{N} \quad h_{D}=\frac{h}{N} \\
w_{x D}=\frac{w_{x}}{N} \quad w_{y D}=\frac{w_{y}}{N} \quad w_{z D}=\frac{w_{z}}{N} \\
x_{0 D}=\frac{x_{0}}{r} \quad y_{0 D}=\frac{y_{0}}{r} \quad z_{0 D}=\frac{z_{0}}{r} \\
x_{D}=\sqrt{\frac{k}{k_{x}}} \frac{x}{N} \quad \begin{array}{c}
y_{D}=\sqrt{\frac{k}{k_{x}}} \frac{y}{N} \quad z_{D}=\sqrt{\frac{k}{k_{x}}} \frac{z}{N} \\
\lambda_{v f}=\frac{\sigma_{v} k_{v} N^{2}}{k_{i f}} \quad \begin{array}{c}
(\phi c)_{j} \\
(\phi c)_{f+m+v}
\end{array}, j=f / m / v \\
\lambda_{m f}=\frac{\sigma_{m} k_{m} N^{2}}{k_{i f}} \\
a_{D}=\frac{a}{N}
\end{array} \quad b_{D}=\frac{b}{N} \\
k_{v}
\end{gathered}
$$




$$
\begin{array}{ccc}
w_{x D}=\frac{w_{x}}{N} & w_{y D}=\frac{w_{y}}{N} & w_{z D}=\frac{w_{z}}{N} \\
x_{0 D}=\frac{x_{0}}{r} & y_{0 D}=\frac{y_{0}}{r} & z_{0 D}=\frac{z_{0}}{r}
\end{array}
$$

Based on the dimensionless transformation above, Equation (4) can be summarized as:

$$
e^{-\Delta p_{f}} \frac{1}{r_{D}^{2}} \frac{\partial}{\partial r_{D}}\left(r_{D}^{2} \frac{\partial \Delta p_{f}}{\partial r_{D}}\right)+\frac{4 \pi}{N^{2}} f\left(r_{D}\right)=\omega_{f} \frac{\partial \Delta p_{f}}{\partial t}+\omega_{m} \frac{\partial \Delta p_{m}}{\partial t}+\omega_{v} \frac{\partial \Delta p_{v}}{\partial t}
$$

Where $f\left(r_{D}\right)$ is

$$
\begin{gathered}
f\left(r_{D}\right)=\frac{1}{8 w_{x D} w_{y D} w_{z D}} \iiint_{v_{D}} \delta\left[\left(r_{D}-r_{0 D}\right)^{3}\right] d v_{D} \\
\left(x_{0 D}-w_{x D}\right)\left(y_{0 D}-w_{y D}\right)\left(z_{0 D}-w_{z D}\right) \leq v_{D} \leq\left(x_{0 D}+w_{x D}\right)\left(y_{0 D}+w_{y D}\right)\left(z_{0 D}+w_{z D}\right)
\end{gathered}
$$

Matrix system dimensionless equation:

$$
-\omega_{m} \frac{\partial \Delta p_{m}}{\partial t_{D}}=\lambda_{m f}\left(\Delta p_{m}-\Delta p_{f}\right)
$$

Cave system dimensionless equation:

$$
-\omega_{v} \frac{\partial \Delta p_{v}}{\partial t_{D}}=\lambda_{v f}\left(\Delta p_{v}-\Delta p_{f}\right)
$$

The internal boundary condition:

$$
\frac{d \Delta p_{f D}}{d t_{D}}-\left.r_{D} \frac{\partial \Delta p_{f D}}{\partial r_{D}}\right|_{r_{D}=1}=1
$$

The outer boundary condition:

$$
\Delta p_{f D}\left(\infty, t_{D}\right)=\Delta p_{v D}\left(\infty, t_{D}\right)=\Delta p_{m D}\left(\infty, t_{D}\right)=0
$$

The initial conditions:

$$
\Delta p_{f D}\left(r_{D}, 0\right)=\Delta p_{v D}\left(r_{D}, 0\right)=\Delta p_{m D}\left(r_{D}, 0\right)
$$

\subsection{Equation Solving}

The Laplace transform of equation (19), (22) and (23) is obtained as follows:

$$
\begin{aligned}
& e^{-\Delta p_{f}} \frac{1}{r_{D}^{2}} \frac{\partial}{\partial r_{D}}\left(r_{D}^{2} \frac{\partial \Delta \overline{p_{f}}}{\partial r_{D}}\right)+\frac{4 \pi}{s N^{2}} f\left(r_{D}\right)=s \omega_{f} \Delta \overline{p_{f}}+s \omega_{m} \Delta \overline{p_{m}}+s \omega_{v} \Delta \overline{p_{v}} \\
& -s \omega_{m} \bar{p}_{m}=\lambda_{m f}\left(\Delta \bar{p}_{m}-\Delta \bar{p}_{f}\right) \\
& -s \omega_{v} \bar{p}_{v}=\lambda_{v f}\left(\bar{p}_{v}-\bar{p}_{f}\right)
\end{aligned}
$$

Substitute equation (28) and (29) into Equation (27), we can get:

$$
e^{-\Delta p_{f}} \frac{1}{r_{D}^{2}} \frac{\partial}{\partial r_{D}}\left(r_{D}^{2} \frac{\partial \Delta \overline{p_{f}}}{\partial r_{D}}\right)+\frac{4 \pi}{s N^{2}} f\left(r_{D}\right)=s f(s) \Delta \overline{p_{f}}
$$


Where

$$
f(s)=\omega_{f}+\omega_{m} \frac{\lambda_{m f}}{\lambda_{m f}+\omega_{m} s}+\omega_{v} \frac{\lambda_{v f}}{\lambda_{v f}+\omega_{v} s}
$$

Equation (30) is a strongly nonlinear partial differential equation, and a perturbation transformation is introduced:

$$
\Delta p_{f}=-\frac{\ln (1-\alpha \psi)}{\alpha}
$$

The derivative of Equation (32) with respect to $r_{\mathrm{D}}$ can be obtained as follows:

$$
\frac{d \Delta p_{f}}{d r_{D}}=\frac{1}{1-\alpha \psi} \frac{d \psi}{d r_{D}}
$$

The derivative of Equation (33) with respect to $t_{\mathrm{D}}$ can be obtained as follows:

$$
\frac{d \Delta p_{f}}{d t_{D}}=\frac{1}{1-\alpha \psi} \frac{d \psi}{d t_{D}}
$$

Substitute equation (33) and (34) into Equation (30), we can get:

$$
\frac{1}{r_{D}^{2}} \frac{\partial}{\partial r_{D}}\left(r_{D}^{2} \frac{\partial \Delta \bar{\psi}}{\partial r_{D}}\right)+\frac{4 \pi}{s N^{2}} f\left(r_{D}\right)=-s f(s) \frac{1}{1-\alpha \psi} \bar{\psi}
$$

In the form of power series, the $\psi$ and $1 /(1-\alpha \psi)$ can be rewritten as

$$
\begin{gathered}
\psi=\psi_{0}+\alpha \psi_{1}+\alpha^{2} \psi_{2}+\alpha^{3} \psi_{3}+\ldots \\
\frac{1}{1-\alpha \psi}=1+\alpha \psi+\alpha^{2} \psi^{2}+\alpha^{3} \psi^{3}+\ldots
\end{gathered}
$$

Because of the small permeability modulus $\psi$, scholars believe that the 0-order perturbation solution can meet the needs of engineering calculation. Take the 0 -order perturbation solution in Equation (36) and Equation (37), and substitute it into Equation (35), we can get:

$$
\frac{1}{r_{D}^{2}} \frac{d}{d r_{D}}\left(r_{D}^{2} \frac{\partial \bar{\psi}_{0}}{\partial r_{D}}\right)+\frac{4 \pi}{s N^{2}} f\left(r_{D}\right)-s f(s) \bar{\psi}_{0}=0
$$

If:

$$
g=r_{D} \bar{\psi}_{0}
$$

Then Equation (38) can be rewritten as: 


$$
\frac{d^{2} g}{d r_{D}^{2}}-s f(s) g=0
$$

The general solution of Equation (40) is

$$
g=A \exp \left(-r_{D} \sqrt{s f(s)}\right)+B \exp \left(r_{D} \sqrt{s f(s)}\right)
$$

Substitute equation (41) into Equation (39), we can get:

$$
\bar{\psi}_{0}=A \frac{\exp \left(-r_{D} \sqrt{s f(s)}\right)}{r_{D}}+B \frac{\exp \left(r_{D} \sqrt{s f(s)}\right)}{r_{D}}
$$

Through internal and external boundary conditions, A and B can be solved:

$$
\begin{gathered}
A=\frac{1}{4 \pi L^{3}} \\
B=0
\end{gathered}
$$

Then, the solution of instantaneous point source in the Laplace space of fracture-vuggy triple medium reservoir is:

$$
\bar{\psi}_{0}=\frac{\mathscr{q}^{\circ}}{\left[\left(\phi c_{t}\right)_{f}+\left(\phi c_{t}\right)_{m}+\left(\phi c_{t}\right)_{v}\right]} \frac{\exp \left(-r_{D} \sqrt{s f(s)}\right)}{4 \pi L^{3} r_{D}}
$$

The above equation is the pressure distribution calculation formula of instantaneous point source function per unit strength, and the point source is located at the origin of coordinates.

\subsection{Discussion}

\section{(1) Point source function is not at the origin of coordinates}

If the point source function is not at the origin of coordinates, but at $\quad \mathrm{x}_{\mathrm{wD}} 、 \mathrm{y}_{\mathrm{wD}}$ 、 $\mathrm{Z}_{\mathrm{wD}}$ ), first define the following expression:

$$
R_{D}=\sqrt{\left(x_{D}-x_{w D}\right)^{2}+\left(y_{D}-y_{w D}\right)^{2}+\left(z_{D}-z_{w D}\right)^{2}}
$$

Then the calculation formula of pressure distribution generated by the unit source function is as follows:

$$
\bar{\psi}_{0}=\frac{\exp \left(-R_{D} \sqrt{s f(s)}\right)}{4 \pi L^{3} R_{D}}
$$

If the instantaneous source function intensity is not 1 , then the pressure calculation formula is as follows: 


$$
\bar{\psi}_{0}=\frac{\mathscr{q}^{\circ}}{\left[\left(\phi c_{t}\right)_{f}+\left(\phi c_{t}\right)_{m}+\left(\phi c_{t}\right)_{v}\right]} \frac{\exp \left(-R_{D} \sqrt{s f(s)}\right)}{4 \pi L^{3} R_{D}}
$$

If:

$$
\bar{S}=\frac{\exp \left(-R_{D} \sqrt{s f(s)}\right)}{4 \pi L^{3} R_{D}}
$$

Then, the time-space solution of Equation (48) is:

$$
\psi_{0}=\frac{q^{\%}}{\left[\left(\phi c_{t}\right)_{f}+\left(\phi c_{t}\right)_{m}+\left(\phi c_{t}\right)_{v}\right]} S\left(t_{D}\right)
$$

By applying the superposition principle, the solution of continuous point source function can be obtained as follows:

$$
\psi_{0}=\frac{1}{\left[\left(\phi c_{t}\right)_{f}+\left(\phi c_{t}\right)_{m}+\left(\phi c_{t}\right)_{v}\right]} \int_{0}^{t} \mathscr{q}(q) S\left(t_{D}-\tau\right) d \tau=\frac{\mu L^{2}}{k_{i f}} \int_{0}^{t_{D}} \mathscr{q}\left(q_{D}\right) S\left(t_{D}-\tau_{D}\right) d \tau_{D}
$$

Based on the property of convolution, Laplace transform of Equation (51), and we can get:

$$
\bar{\psi}_{0}=\frac{\bar{q} / \phi}{4 \pi k_{i f} L} \frac{\exp \left(-R_{D} \sqrt{s f(s)}\right)}{R_{D}}
$$

\section{(2) Upper and lower closed boundary}

The pressure of the fracture-vuggy reservoir is solved based on the mirror image principle. It is assumed that the point source is located in a plate fracture-vuggy reservoir with closed upper and lower boundaries. The reservoir height is $h$. The position of the point source is $z_{w}$. Schematic diagram of closed boundary mirroring principle, as shown in figure 3.

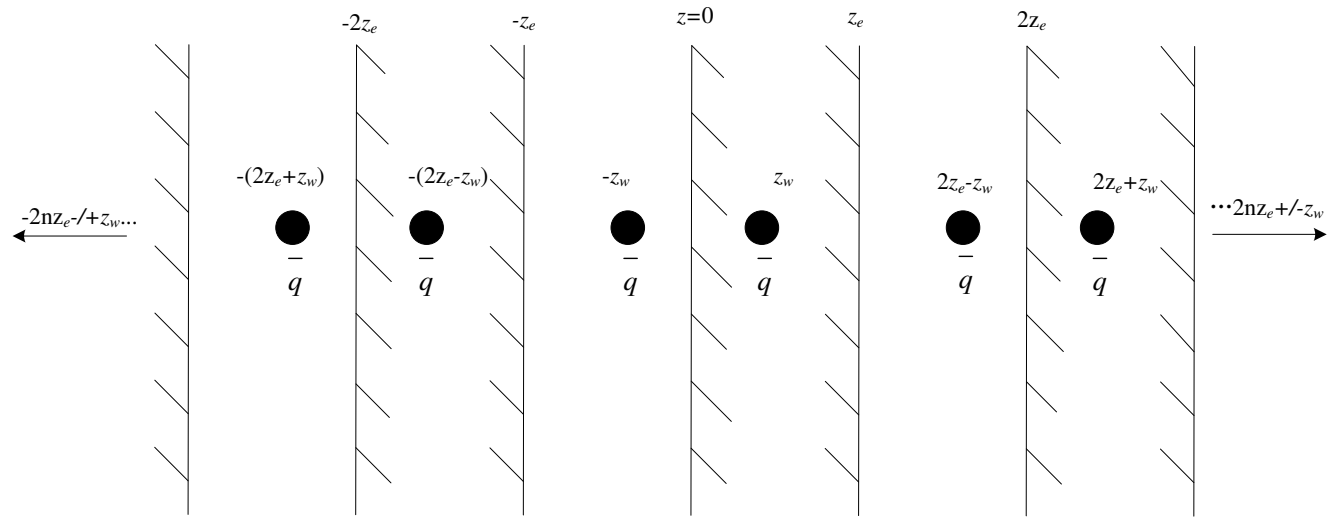

Figure 3 Schematic diagram of mirroring principle of closed boundary reservoir 
After mirror inversion, the position of the point source is:

$$
\left\{\begin{array}{l}
2 n h_{D}-z_{D w} \\
2 n h_{D}+z_{D w}
\end{array} \quad(n=0, \pm 1, \pm 2, \pm 3, \ldots, \pm \infty)\right.
$$

Based on the superposition principle, the point source function of upper and lower closed plate fracture-vuggy reservoir can be expressed as:

$$
\begin{aligned}
\bar{\psi}_{0}= & \frac{\bar{q} / \phi}{4 \pi k_{f} L} \sum_{n=-\infty}^{\infty}\left\{\frac{\exp \left(-\sqrt{s f(s)} \sqrt{\left(x_{D}-x_{w D}\right)^{2}+\left(y_{D}-y_{w D}\right)^{2}+\left(z_{D}+z_{D w}-2 n h_{D}\right)^{2}}\right)}{\sqrt{\left(x_{D}-x_{w D}\right)^{2}+\left(y_{D}-y_{w D}\right)^{2}+\left(z_{D}+z_{D w}-2 n h_{D}\right)^{2}}}\right. \\
& \left.+\frac{\exp \left(-\sqrt{s f(s)} \sqrt{\left(x_{D}-x_{w D}\right)^{2}+\left(y_{D}-y_{w D}\right)^{2}+\left(z_{D}-z_{D w}-2 n h_{D}\right)^{2}}\right)}{\sqrt{\left(x_{D}-x_{w D}\right)^{2}+\left(y_{D}-y_{w D}\right)^{2}+\left(z_{D}-z_{D w}-2 n h_{D}\right)^{2}}}\right\}
\end{aligned}
$$

This equation is not convenient to solve. Possion relation, trigonometric function and difference product formula can be used to transform the above equation, and the following equation can be obtained:

$$
\bar{\psi}_{0}=\frac{\bar{q} / \phi}{2 \pi k_{f} L h_{D}}\left[K_{0}\left(r_{D} \sqrt{s f(s)}\right)+2 \sum_{n=1}^{\infty} K_{0}\left(r_{D} \sqrt{s f(s)+\left(\frac{n \pi}{h_{D}}\right)^{2}}\right) \cos n \pi \frac{z_{D}}{h_{D}} \cos n \pi \frac{z_{w D}}{h_{D}}\right]
$$

\section{(3) Upper and lower constant pressure boundaries}

Similarly, when the upper and lower boundaries have constant pressure, the solution of the point source function is:

$$
\bar{\psi}_{0}=\frac{\bar{q} / \mu}{\pi k_{f} L h_{D}}\left[\sum_{n=1}^{\infty} K_{0}\left(r_{D} \sqrt{s f(s)+\left(\frac{n \pi}{h_{D}}\right)^{2}}\right) \sin n \pi \frac{z_{D}}{h_{D}} \sin n \pi \frac{z_{w D}}{h_{D}}\right]
$$

When $\mathrm{z}=0$ is the closed boundary, and $\mathrm{z}=\mathrm{h}$ is the constant pressure boundary, the solution of the point source function is:

$$
\bar{\psi}_{0}=\frac{\bar{q} / \mathscr{P}}{\pi k_{f} L h_{D}}\left[\sum_{n=1}^{\infty} K_{0}\left(r_{D} \sqrt{s f(s)+\frac{(2 n-1)^{2} \pi^{2}}{4 h_{D}^{2}}}\right) \cos (2 n-1) \frac{\pi}{2} \frac{z_{D}}{h_{D}} \cos (2 n-1) \frac{\pi}{2} \frac{z_{w D}}{h_{D}}\right]
$$

The boundary types of fracture-cavity reservoirs are diverse, so we will not discuss them one by one here.

Based on the above three types of point source functions with different upper and lower boundary properties, seepage solution equations can be constructed under different conditions. For the physical model in this article, the length of the horizontal 
section of the horizontal well is L, and the pressure solving steps are as follows:

(1) Firstly, it is assumed that the length and height of a certain fracture are $2 L_{\mathrm{fi}}$ and $h_{\text {wi. }}$ Then, according to the boundary type of fracture-vuggy reservoir (the above pressure boundary is taken as an example to illustrate), Formula (57) integrates $\mathrm{x}$ in the interval of $\left(\mathrm{x}_{\mathrm{w}}-\mathrm{L}_{\mathrm{f}}, \mathrm{x}_{\mathrm{w}}+\mathrm{L}_{\mathrm{f}}\right)$, and then integrates $\mathrm{z}$ in the interval of $\left(\mathrm{z}_{\mathrm{w}}-\mathrm{h}_{\mathrm{w}} / 2, \mathrm{z}_{\mathrm{w}}+\mathrm{h}_{\mathrm{w}} / 2\right)$. The expression of the pressure of a single fracture in the horizontal section of the horizontal well can be obtained.

(2) According to the superposition principle, the pressure generated by each fracture in the corresponding horizontal section is superimposed to obtain the Laplace space solution of the total pressure.

(3) According to the solution of Laplace space, the Stehfest method is used to carry out numerical inversion, and the numerical solution of real space is obtained.

\section{Model Validation}

\subsection{Literature comparison and validation}

Riley et al. solved the analytical solution of bottom hole pressure in horizontal wells with only one fracture supply. For the convenience of comparison, it is assumed that the fracture in the physical model of horizontal well in this paper is one. The angle between the fracture and the horizontal section is $90^{\circ}$. The fracture locates in the center of the horizontal wellbore. To be consistent with the conditions in the literature, the influence of karst caves is not considered. Other basic reservoir parameters are shown in table 1. The calculation results of Riley et al. and those in this paper are shown in figure 4. Figure 4 shows that the results of the two have a good consistency. Riley et al. is a special case of the model in this paper, so the model in this paper is correct.

\begin{tabular}{|c|c|c|c|}
\hline parameter & value & parameter & value \\
\hline original saturation pressure $(\mathrm{MPa})$ & 44 & effective thickness (m) & 30 \\
\hline formation oil viscosity (mPa.s) & 0.45 & horizontal length & 400 \\
\hline original reservoir pressure $(\mathrm{MPa})$ & 65 & crude oil density $\left(\mathrm{g} / \mathrm{cm}^{3}\right)$ & 0.729 \\
\hline number of fractures & 1 & comprehensive compression coefficient $\left(10^{-4} 1 / \mathrm{MPa}\right)$ & 13.8 \\
\hline
\end{tabular}




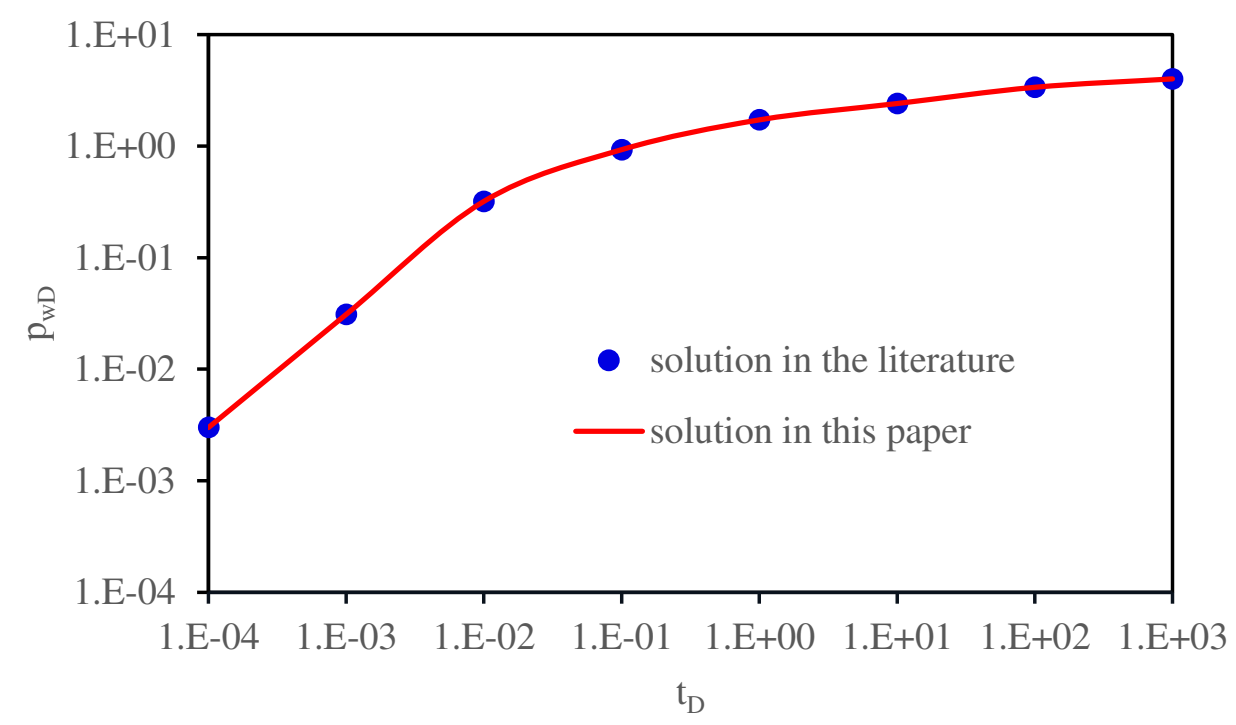

Figure 4 Comparison of calculation results

\subsection{The numerical simulation validation}

For comparison purposes, the number of fractures is assumed to be 5 . The relevant parameters of fractures are shown in table 2. The reservoir is a plate reservoir with upper and lower boundary with constant pressure. By simulating the productivity of horizontal wells with different fracture numbers and fracture locations, the bottom hole pressure of the corresponding horizontal wells (the simulation solution) was obtained and compared with the pressure obtained from numerical inversion in this paper (this paper solution).

Table 2 List of fracture parameters

\begin{tabular}{|l|c|c|c|c|c|}
\hline parameter & fracture 1 & fracture 2 & fracture 3 & fracture 4 & fracture 5 \\
\hline location & $(500,500)$ & $(550,500)$ & $(600,500)$ & $(650,500)$ & $(700,500)$ \\
\hline angle with horizontal segment & $30^{\circ}$ & $45^{\circ}$ & $60^{\circ}$ & $75^{\circ}$ & $90^{\circ}$ \\
\hline fracture width & $10 \mathrm{~mm}$ & $8 \mathrm{~mm}$ & $6 \mathrm{~mm}$ & $4 \mathrm{~mm}$ & $2 \mathrm{~mm}$ \\
\hline fracture half-length & $200 \mathrm{~m}$ & $160 \mathrm{~m}$ & $120 \mathrm{~m}$ & $80 \mathrm{~m}$ & $40 \mathrm{~m}$ \\
\hline fracture height & $5 \mathrm{~m}$ & $5 \mathrm{~m}$ & $5 \mathrm{~m}$ & $5 \mathrm{~m}$ & $5 \mathrm{~m}$ \\
\hline initial horizontal permeability & $100 \mathrm{mD}$ & $100 \mathrm{mD}$ & $100 \mathrm{mD}$ & $100 \mathrm{mD}$ & $100 \mathrm{mD}$ \\
\hline initial vertical permeability & $500 \mathrm{mD}$ & $500 \mathrm{mD}$ & $500 \mathrm{mD}$ & $500 \mathrm{mD}$ & $500 \mathrm{mD}$ \\
\hline
\end{tabular}

The E300 module in Eclipse 2017 is designed for triple-media fracture-vuggy reservoirs. To meet the assumptions of the model derived in this paper, the numerical model is set as follows: The width and length of the heterogeneous fracture-vuggy reservoir are $1000 \mathrm{~m}$, and there is a horizontal well in the center of the reservoir with a horizontal section length of $200 \mathrm{~m}$, as shown in figure $5(\mathrm{a})$. The triangulation mesh 
method is adopted to divide meshing and ensure that each fracture has at least three meshings to describe the heterogeneity of formation fluid. If the fracture is short, local mesh encryption should be performed at the fracture, as shown in figure 5(b). The plane mesh step size is $20 \mathrm{~m}$, and the vertical mesh step size is $5 \mathrm{~m}$. Then the total number of grids is $50 * 50 * 16=40000$. Other parameters required for numerical simulation are shown in table 3 . The numerical simulation and calculation results in this paper are shown in table 4 . Table 4 shows that the relative error is controlled within $5 \%$ under the basic error, which is consistent with the allowable error range, indicating that the method we provide is reliable.

\begin{tabular}{|c|c|c|c|}
\hline Table 3 basic reservoir parameters & parameter & value \\
\hline parameter & value & formation water density $\left(\mathrm{g} / \mathrm{cm}^{3}\right)$ & 1.0 \\
\hline original saturation pressure (MPa) & 44 & formation oil volume coefficient & 1.612 \\
\hline formation oil viscosity (mPa.s) & 0.45 & crude oil density $\left(\mathrm{g} / \mathrm{cm}^{3}\right)$ & 0.729 \\
\hline permeability modulus (1/MPa) & 0.0012 & oil-water interface depth (m) & 3100 \\
\hline surface oil viscosity (mPa.s) & 1.71 & formation water volume coefficient & 1.102 \\
\hline formation water viscosity (mPa.s) & 0.50 & formation oil compression coefficient & 34 \\
\hline coefficients of compression of rock & 5.0 & (10-41/MPa) & 34 (MPa) \\
\hline horizontal well length(m) & 200 & coefficients of compression of formation & 4.2 \\
\hline formation thickness(m) & 80 & fracture storage capacity ratio & 0.15 \\
\hline initial formation pressure(Mpa) & 65 & cave storage volume ratio & 0.25 \\
\hline number of fractures & 5 & matrix storage capacity ratio & 0.60 \\
\hline radial permeability(mD) & 30 & porosity & 0.15 \\
\hline
\end{tabular}

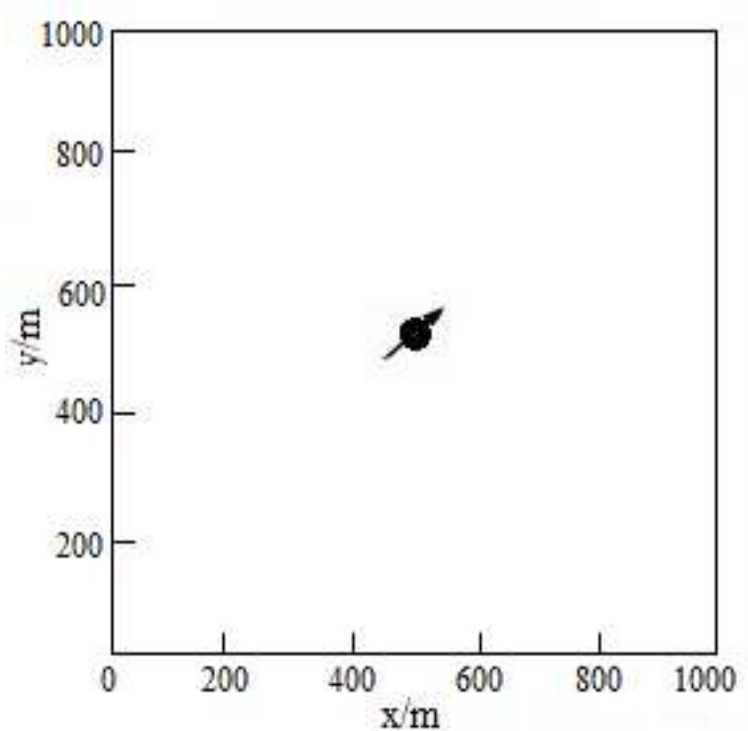

(a) well location

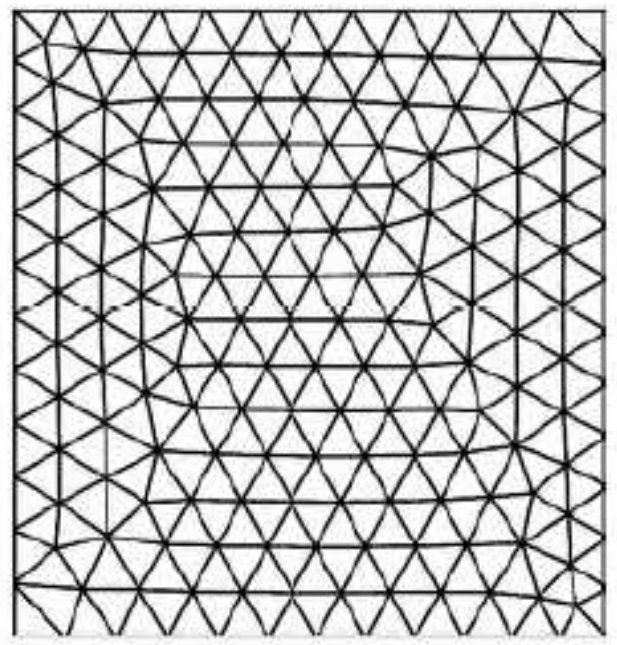

(b) grid configuration

Figure 5 Schematic diagram of well location and grid division 
Table 4 Comparison of calculation results

\begin{tabular}{|c|c|c|c|c|}
\hline $\begin{array}{c}\text { production time } \\
(\mathrm{d})\end{array}$ & $\begin{array}{c}\text { productivity } \\
\left(\mathrm{m}^{3} / \mathrm{d}\right)\end{array}$ & $\begin{array}{c}\text { the simulation } \\
\text { solution }(\mathrm{MPa})\end{array}$ & $\begin{array}{c}\text { this paper solution } \\
(\mathrm{MPa})\end{array}$ & relative error (\%) \\
\hline 30 & 33.137992 & 51.807017 & 50.148556 & 0.032012285 \\
\hline 60 & 32.651338 & 50.204722 & 48.549205 & 0.032975325 \\
\hline 90 & 32.164684 & 48.963757 & 47.317118 & 0.033629752 \\
\hline 120 & 31.67803 & 47.962682 & 46.336766 & 0.033899606 \\
\hline 150 & 30.91002 & 47.130427 & 45.52229 & 0.034120994 \\
\hline 180 & 30.89413 & 46.292744 & 44.474019 & 0.039287475 \\
\hline 210 & 30.68332 & 45.876605 & 43.841381 & 0.044363004 \\
\hline 240 & 29.35811 & 44.004106 & 42.051935 & 0.044363383 \\
\hline 270 & 27.86354 & 43.377195 & 41.2114805 & 0.049927491 \\
\hline 300 & 25.92476 & 42.3976848 & 41.8508976 & 0.012896629 \\
\hline 330 & 22.11243 & 41.4181746 & 40.6985112 & 0.017375546 \\
\hline 360 & 19.15212 & 40.4386644 & 39.5461248 & 0.022071441 \\
\hline
\end{tabular}

\subsection{Flow Characteristics Analysis}

A fracture-vuggy reservoir with a rectangular outer boundary has a horizontal well in the center. The number of fractures is 4 . The length of the horizontal section of the horizontal well is $400 \mathrm{~m} . k_{\mathrm{x}}=k_{\mathrm{y}}, k_{\mathrm{z}} / k_{\mathrm{x}}=100$. The wellbore storage factor is 0.001 , and the skin factor is 1 . Based on the basic parameters in Table 1, the dimensionless pressure and pressure derivative were calculated, as shown in figure 6. Figure 6 shows that the fluid flow process of the triple media fracture-vuggy reservoir can be divided into five stages, considering fracture orientation and stress sensitivity.

Stage A: The wellbore storage phase. The pressure and pressure derivative curves coincide, and the asymptotic analysis shows that the slope of the curve is about 1. This stage is mainly affected by the wellbore storage effect.

Stage B: The karst cave fluid flows to the fracture stage. Generally, the permeability of the karst cave system is greater than that of the fracture system. As the fracture system supplies fluid to the wellbore, the pressure of the fracture system drops. Then the karst cave system first supplies fluid to the fracture to supplement the pressure of the fracture system. A "dent" appears in the curve.

Stage C: Radial flow stage of cave and fracture system. The slope of the pressure derivative curve is about 0.5 when the fracture fluid supplied by the cave system is in equilibrium with the wellbore fluid supplied by the fracture system. This stage is short or infrequent due to the presence of a matrix, which also supplies fracture 
fluid.

Stage D: Matrix fluid flows to the fracture stage. When the fluid in the cave and fracture system is extracted, the pressure of the two systems decreases continuously until it is less than the pressure of the matrix system, and the fluid in the matrix begins to flow to the fracture and cave under the action of pressure difference.

Stage E: Quasi-steady state stage. When the flow between matrix - fracture, cave - fracture, and fracture - wellbore is in equilibrium, the system reaches the quasi-steady state. The pressure derivative curve approximates a horizontal line, and the asymptotic analysis shows that the horizontal line is about 0.5 .

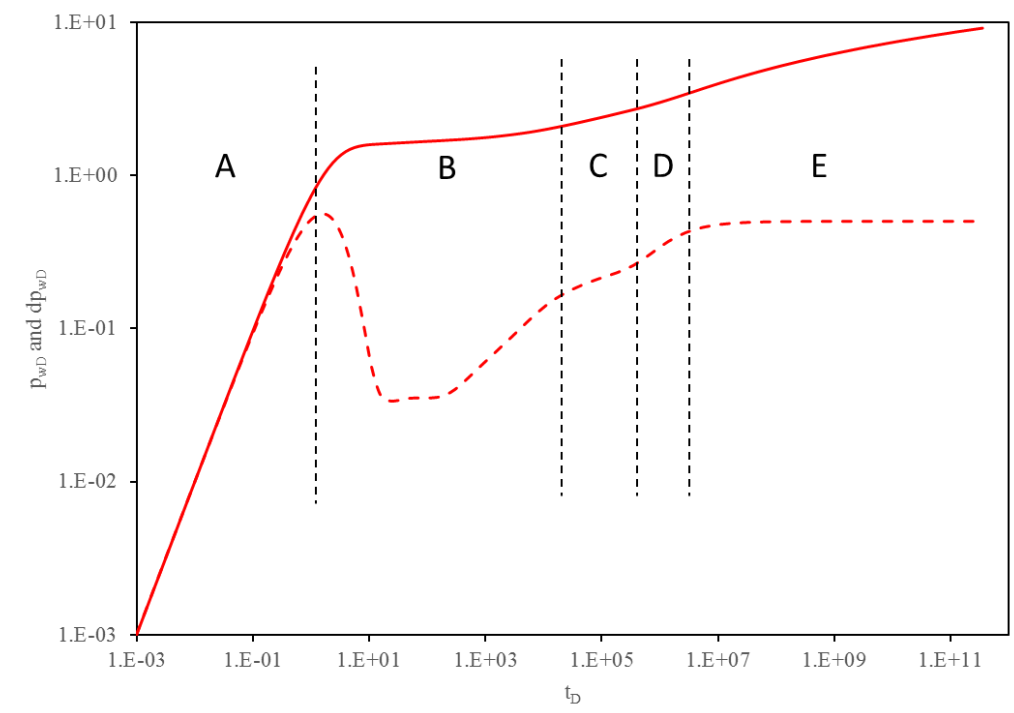

Figure 6 The flow division schematic of the type curves (Solid line: $p_{\mathrm{wD}}$ Dash line: $\mathrm{d} p_{\mathrm{wD}}$ )

\section{Sensitivity analysis}

According to the method and process of solving bottom hole pressure in this paper, the sensitivity analysis of the influencing factors of pressure and pressure derivative is carried out based on the principle of the single factor variable. The influences of fracture number, fracture angle, fracture half-length, skin factor, and horizontal well segment length, and horizontal well segment spacing on pressure and pressure derivative are analyzed in detail.

\subsection{Horizontal segment spacing of horizontal well}

When the total length of the reservoir shot by the horizontal well is fixed, the entire drainage area communicating with the fractures outside the wellbore is certain. The influence of horizontal segment spacing on the type curves is shown in figure 7. Figure 7 shows that horizontal segment spacing mainly affects stages B and $\mathrm{C}$. The larger the 
interval between the horizontal segments of the horizontal well, the longer it takes for fractures to supply the wellbore. The more difficult the channeling flow between the cave and fracture will be.

When the horizontal segment spacing is large, a new "platform" appears on the pressure derivative curve in stage $\mathrm{C}$, with a horizontal line of about 0.25 . The asymptotic analysis shows that the new "platform" height depends on the number of segments in the horizontal well. In general, when the number of segments in the horizontal well is $\mathrm{n}$, the corresponding horizontal line of the new "platform" is about $1 / \mathrm{n}$.

Infield well testing, it isn't easy to have stage $\mathrm{E}$ if the test time is not long enough. Therefore, during well test interpretation, the actual permeability can be obtained by multiplying the permeability $\mathrm{k}$ explained in stage $\mathrm{C}$ by the number of segments of horizontal wells.

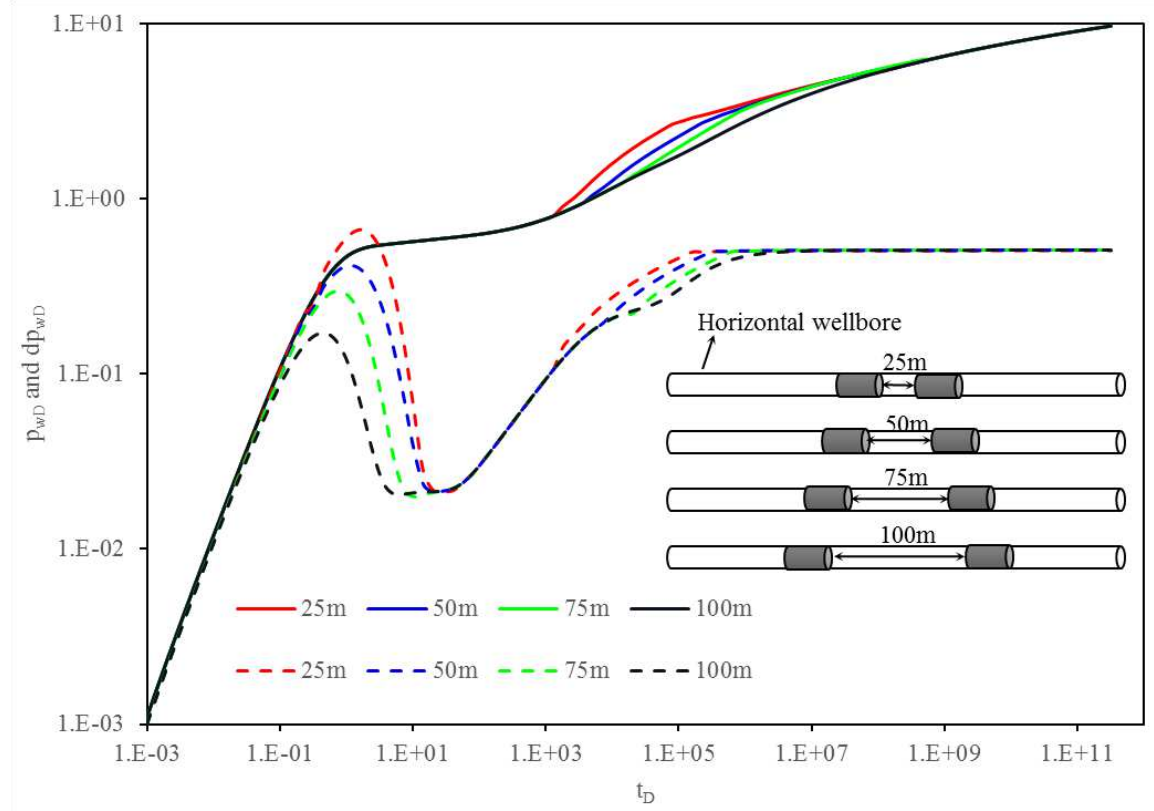

Figure 7 The effect of segment spacing on type curves (Solid line: $p_{\mathrm{wD}}$ Dash line: $\mathrm{d} p_{\mathrm{wD}}$ )

\subsection{Horizontal well segment length}

The influence of different horizontal segment lengths on the type curves is shown in figure 8 . Figure 8 shows that segment length mainly affects stage B. The longer the segment length is, the lower the "hump" is, and the earlier stage B is. However, the shorter the duration of stage $\mathrm{B}$ is, and the lower the value of the pressure derivative is and more quickly recovered to 0.5 . The larger the reservoir interval opened by horizontal wells, the more favorable the flow of reservoir fluid to the wellbore, and the earlier the well reservoir effect will disappear. 


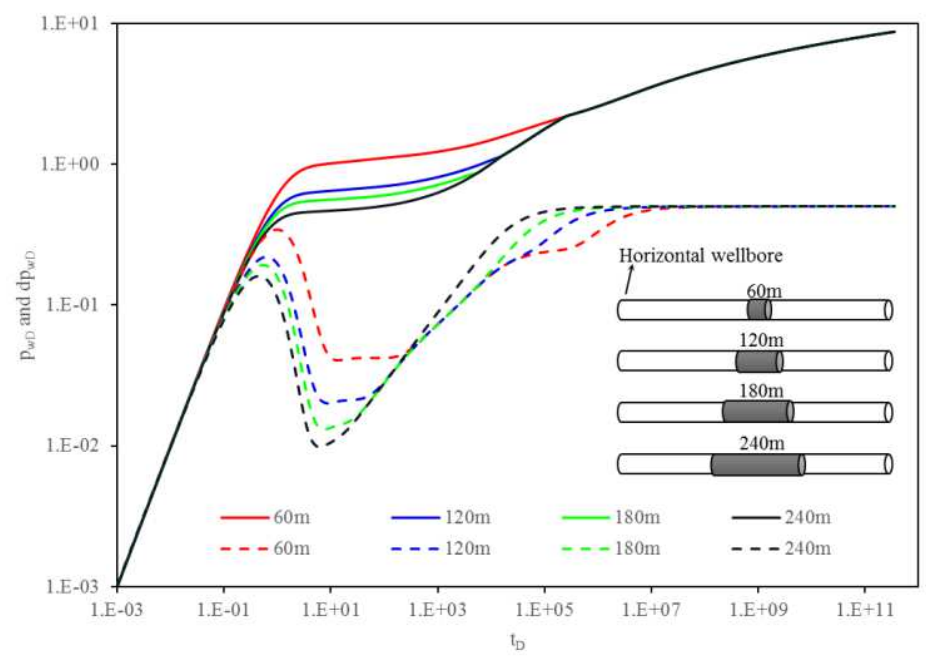

Figure 8 The effect of section length on type curves (Solid line: $p_{\mathrm{wD}}$ Dash line: $\mathrm{d} p_{\mathrm{wD}}$ )

When the total length of the horizontal segment is fixed, the influence of each segment's length and distribution mode on the type curves is shown in figure 9. Figure 9 shows that when the section length at both ends of the horizontal well increases, the pressure drop generated becomes smaller, conducive to horizontal well production. Therefore, in the actual perforation process, it is suggested to increase the production section at the toe and foot ends of the horizontal well to increase the supply of fractures to the horizontal wellbore and improve the horizontal wellbore productivity.

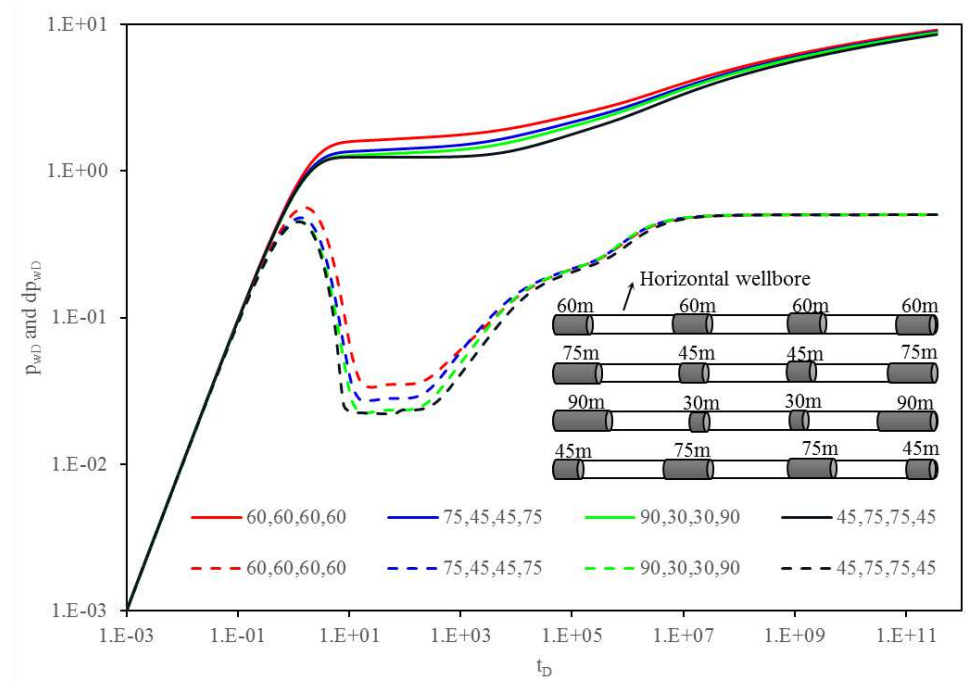

Figure 9 The effect of section length and distribution mode on type curves (Solid line: $p_{\mathrm{wD}}$. Dash line:d $p_{\mathrm{wD}}$ )

\subsection{Skin factor}

The influence of the skin factor on the type curves is shown in figure 10 . Figure 10 shows that the larger the skin factor is, the more serious the wellbore pollution is. What's more, the longer the duration of stage $\mathrm{A}$ is, and the later the appearance of stage $\mathrm{B}$ is, and the higher the "hump" is. Stage $\mathrm{C}$ is covered in varying degrees, and the skin factor affects the radial flow of the fracture. 


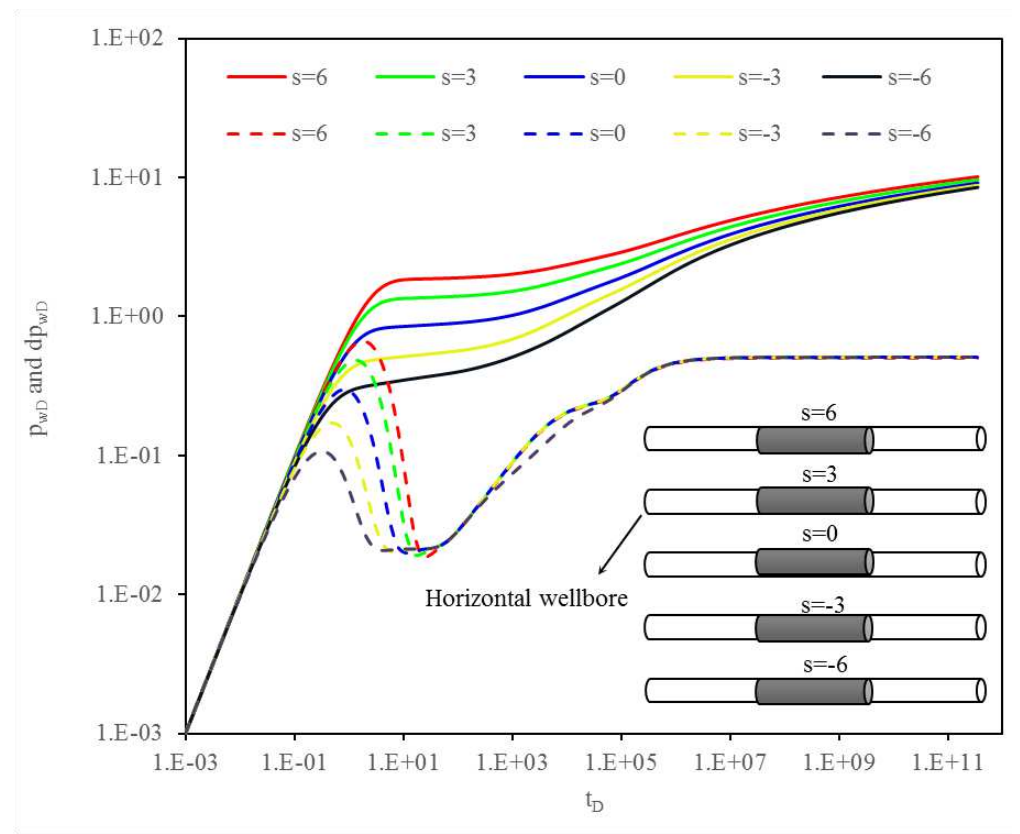

Figure 10 The effect of skin factor on type curves (Solid line: $p_{\mathrm{wD}}$. Dash line:d $p_{\mathrm{wD}}$ )

When the sum of the total skin factor of each segment is constant, the influence of the size and distribution of the skin factor of each segment on the type curves is shown in figure 11. Figure 11 shows that the skin factor causes different flow rates in each section, and the non-uniformly distributed skin factor produces different pressure responses at the bottom of the well.

When the pollution at both ends of the horizontal well is relatively serious, the pressure drop loss caused is greater, which is not conducive to improving the production of the horizontal well. Therefore, when using horizontal wells to implement reservoir stimulation measures, special attention should be paid to modifying the formations at both ends of the horizontal well to eliminate pollution near the wellbore.

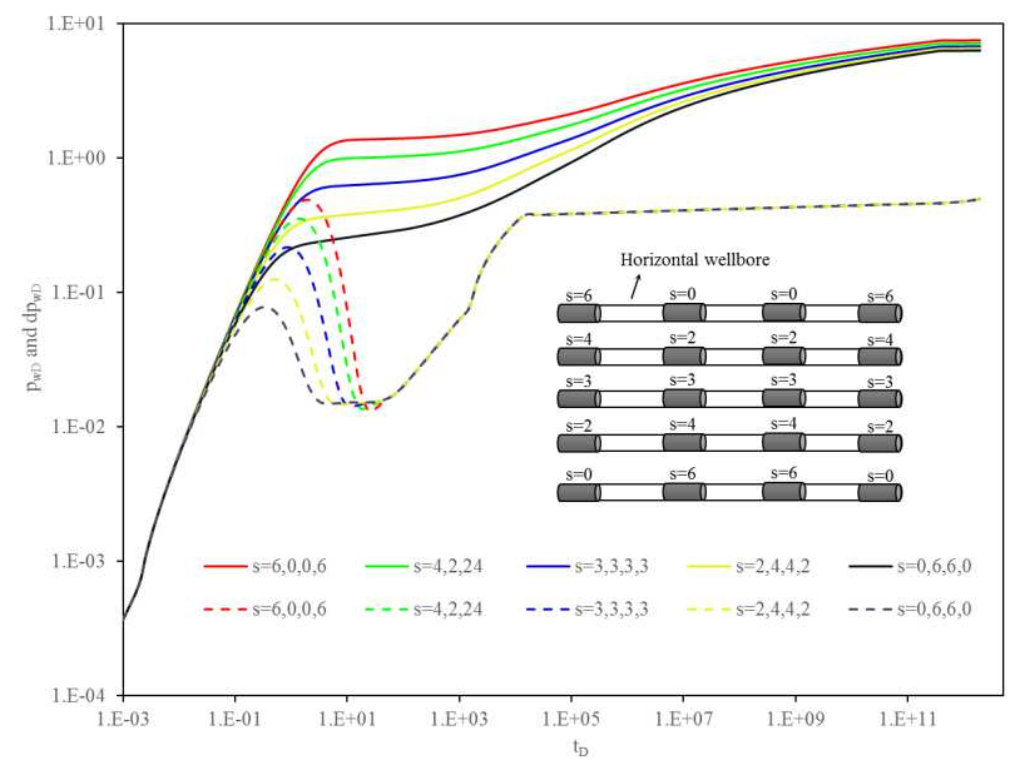

Figure 11 The effect of size and distribution of skin factor on type curves (Solid line: $p_{\mathrm{wD}}$. Dash line:d $p_{\mathrm{wD}}$ ) 


\subsection{Fracture number}

The influence of the fracture number on the type curves is shown in figure 12 . Figure 12 shows that the fracture number mainly affects stage $\mathrm{C}$. When there are many fractures, and the fractured-vuggy reservoir has a fracture network, stage D will be covered by stage $\mathrm{C}$. To a certain extent, the matrix does not directly supply the caves. Instead, the matrix directly supplies the fractures, mainly caused by the development of a fracture network in the reservoir. The more developed the fracture network, the seepage characteristics of the triple-medium fracture-cavity reservoir are more similar to the dual-media. The matrix/cavities in the reservoir directly supply the fractures and then flow to the wellbore. This flow mode is more conducive to pressure transmission and production increase.

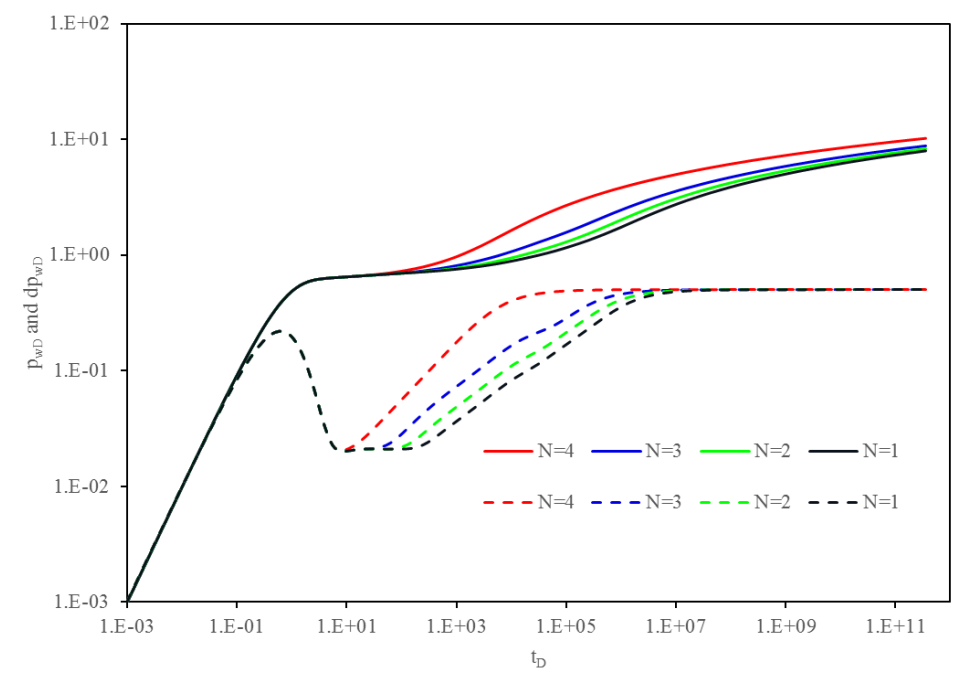

Figure 12 The effect of the fracture number on type curves (Solid line: $p_{\mathrm{wD}}$. Dash line: $\mathrm{d} p_{\mathrm{wD}}$ )

\subsection{Fracture direction}

When the angle between the fracture and the horizontal wellbore changes, different fracture angles have different effects on the type curves, as shown in Figure 13. Figure 13 shows that the fracture direction mainly affects stage $C$. When the fracture is perpendicular to the wellbore, the pressure drop is smaller, and the pressure transmission is faster, which is more conducive to improving the productivity of horizontal wells. Because the drainage area is larger, and more fluid in the reservoir flows through the fractures to the wellbore at the same time. The perforating gun should be parallel to the horizontal wellbore during fracturing as much as possible, and then fractures perpendicular to the wellbore should be generated. 


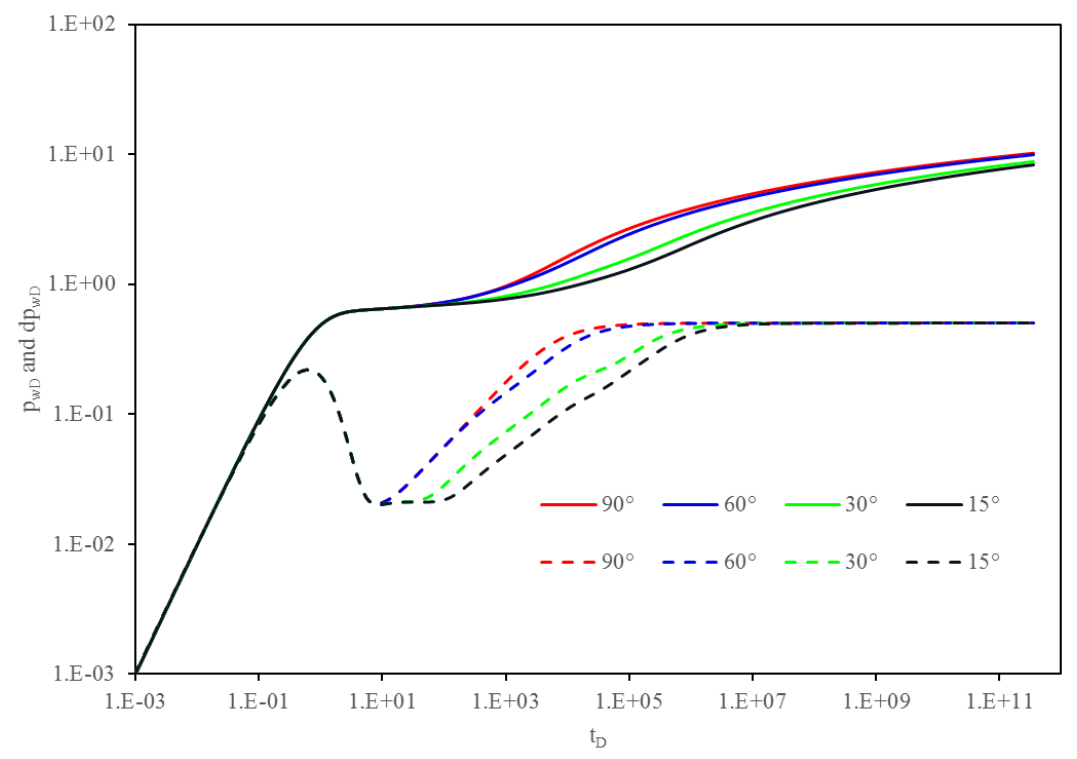

Figure 13 The effect of the fracture direction on type curves (Solid line: $p_{\mathrm{wD}}$. Dash line: $\mathrm{d} p_{\mathrm{wD}}$ )

\subsection{Fracture half-length}

The effect of the fracture half-length on the type curves is shown in figure 14. Figure 14 shows that the fracture half-length mainly affects stage B and stage $\mathrm{C}$. When the fracture half-length is longer, the pressure drop is smaller, which is most conducive to production. When the fracture half-length is very small $(\leq 50 \mathrm{~m})$, stage D is more obvious because the fracture half-length is small, the fluid in the matrix cannot flow directly to the fracture. It has to flow to the cave first and then flows to the fracture through the cave. The drainage area in the reservoir is limited, and the pressure spreads more slowly. Therefore, in the design of hydraulic fracturing, the fracture half-length should be greater than $50 \mathrm{~m}$ as far as possible.

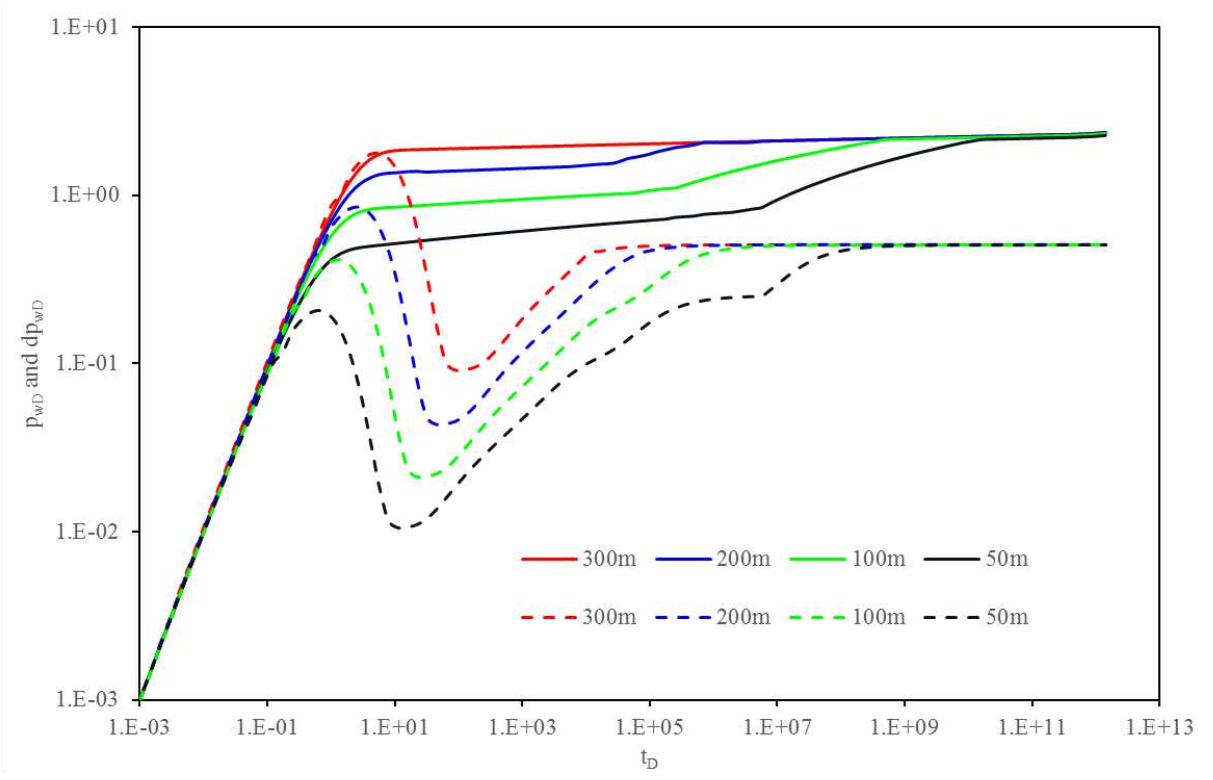

Figure 14 The effect of fracture half-length on type curves (Solid line: $p_{\mathrm{wD}}$. Dash line: $\mathrm{d} p_{\mathrm{wD}}$ ) 


\section{Conclusion}

In this paper, a new point source function for triple-medium fractured-vuggy reservoirs is established, and the steps for solving the bottom hole pressure of horizontal wells are given. Through literature comparison and numerical simulation methods, the rationality of the method in this paper is verified. The influence of fracture number, fracture angle, fracture half-length, skin factor, horizontal well segment length, and horizontal well segment spacing on pressure and pressure derivative is analyzed in detail. The study believes special attention should be paid to reforming the formations at both ends of the horizontal well to eliminate pollution near the wellbore when using horizontal wells to implement reservoir production stimulation measures. When designing pressure construction, the perforating gun should be made parallel to the horizontal wellbore as much as possible, and the fracture half-length formed should be greater than $50 \mathrm{~m}$. When designing horizontal well fracturing, it is necessary to increase the production section at the toe and toe ends of the horizontal well, expand the oil drainage area, and accelerate the supply of fractures to the horizontal wellbore to achieve the purpose of increasing the productivity of the horizontal well.

\section{Conflict of Interests}

The authors declare that there is no conflict of interests regarding the publication of this paper.

\section{References}

[1] J E Warren, P J Rot. The behavior of Naturally Fractured Reservoirs[J].SPE 1842,1963 .

[2] H J Ramey, A C Gringarten. The use of source and Green's function in solving unsteady-flow problem in reservoir[J].SPE 3818,1973.

[3] H J Ramey, A C Gringarten. Unsteady Pressure Distribution Created by a Single Horizontal Fracture, and Partial Penetration, or Restricted Entry[R].SPE 3819,1974.

[4] F J Kuchuk, P A Kirwan.New Skin and Well bore Storage Type Curves for Partially Penetrated Wells[R].SPE 4676,1987.

[5] Brown M, Ozkan E, Raghavan R, etal. Practical Solutions for Pressure Transient Responses of Fractured Horizontal Wells in Unconventional Reservoirs[C]. SPE 4782,1987 
[6] M Onur, A Satman. New Type Curve for Analyzing the Transition Time Data from Naturally Fractured Reservoirs[R].SPE 25873,1993.

[7] Zhang Xuewen. Study on steady state solution productivity formula of fractured vertical well in low permeability reservoir $[\mathrm{J}]$. Fault Block Oil \& Gas Field,1999,6(1):48-51

[8] T D Bui, D D Mamora, W J Lee. Transient Pressure Analysis for Partially Penetrating Wells in Naturally Fractured Reservoirs[R].SPE 60289,2000.

[9]Song Fuquan, Liu Ciqun. Pressure production analysis method for deformable media reservoirs[J]. Petroleum Exploration and Development, 2001, 27(1): 57-59.

[10] Deng Yinger, Liu Ciqun. Analysis of non-linear seepage pressure in the development of low-permeability reservoirs with vertical fractured wells[J]. Petroleum Exploration and Development, 2003, 30(1): 81-83

[11] Li Sheng, Li Xia, Zeng Zhilin, etc. Productivity evaluation of vertical fractured wells in tight sandstone reservoirs[J]. Daqing Petroleum Geology and Development, 2005, 24(1): 54-58.

[12]Zhang Nan, Wang Xiaoqin, Xu Feng, etc. The influence of starting pressure gradient and stress sensitivity on productivity of vertical wells in tight sandstone reservoirs[J]. Fault Block Oil and Gas Field, 2008, 19(1): 74-79.

[13] Zhang Xiuan, Guo Xiao, Yang Kai, etc. The influence of threshold pressure gradient and stress sensitivity on productivity of tight sandstone reservoirs[J]. Journal of Chongqing University of Science and Technology (Natural Science Edition), 2010, 12(2): 49- 51.

[14] Zhang Xiaoliang, Li Di, Sun Junshu, etc. Productivity analysis of fractured wells in tight sandstone reservoirs[J]. Science and Technology Review, 2011, 29(19): 58-61.

[15] Stalgorova E, Matter L. Practical Analytical Model Simulate Production of Horizontal Wells with Branch Fractures[C]. SPE 327851,2012.

[16] Stalgorova K, Mattar L. Analytical Model for Unconventional Multifractured Composite Systems[J]. SPE Reservoir Evaluation \& Engineering, 2013, 16(3):246-256. [17] Jiang Ruizhong, Yang Ming, Wang Gongchang, etc. Production performance analysis of fractured wells in tight sandstone reservoirs[J]. Special Oil and Gas 
Reservoirs, 2013, 20(1): 51:56.

[18] Wang Feng, Liu Huiqing, Lu Guangzhong. Steady-state productivity prediction model for long fractured vertical wells in tight sandstone reservoirs[J]. Petroleum Geology and Recovery Efficiency, 2014, 21(1): 84-90.

[19] Zhang Tiantian, Duan Yonggang, Wei Mingqiang. Research on productivity evaluation and fracture parameter optimization of fractured wells in tight sandstone reservoirs[J]. Reservoir Evaluation and Development, 2015, 5(6): 27-31.

[20] Sureshjani M H, Clarkson C R. An Analytical Model for Analyzing and Forecasting Production from Multifractured Horizontal Wells with Complex Branched-Fracture Geometry[J]. SPE Reservoir Evaluation \& Engineering, 2015, 18(3):182-191

[21] Hailong,Liu. The numerical simulation for multistage fractured horizontal well in low-permeability reservoirs based on modified Darcy's equation[J]. J Petrol Explor Prod Technol (2016). doi:10.1007/s13202-016-0283-1.

[22] Stehfest H. Numerical Inversion of Laplace Transform [R].ACM,1970,20(1):47-48. [23] M. F. Riley,W. E. Brigham, and R. N.Horne, "Analytic solutions for elliptical finite-conductivity fractures," in Proceedings of the SPE Annual Technical Conference and Exhibition, pp. 35-48, October 1991, SPE paper no. 22656. 